\title{
Artificial Intelligence: A New Tool in Oncologist's Armamentarium
}

\author{
Vineet Talwar ${ }^{1} \quad$ Kundan Singh Chufal $^{2}$ Srujana Joga ${ }^{1}$ \\ ${ }^{1}$ Department of Medical Oncology, Rajiv Gandhi Cancer Institute \& \\ Address for correspondence Srujana Joga, DNB General Medicine, \\ Research Centre, New Delhi, India \\ 2 Department of Radiation Oncology, Rajiv Gandhi Cancer Institute \& \\ Research Centre, New Delhi, India \\ Department of Medical Oncology, Rajiv Gandhi Cancer Institute \& \\ Research Centre, Rohini, Sector 5, New Delhi, 110085, India \\ (e-mail: srujanajoga@gmail.com).
}

Ind J Med Paediatr Oncol 2021;42:511-517.

\begin{abstract}
\section{Keywords}

- artificial intelligence

- oncology

- radiation oncology

- translational oncology

- applications

- clinical decision

- clinical outcomes

Artificial intelligence (Al) has become an essential tool in human life because of its pivotal role in communications, transportation, media, and social networking. Inspired by the complex neuronal network and its functions in human beings, Al, using computer-based algorithms and training, had been explored since the 1950s. To tackle the enormous amount of patients' clinical data, imaging, histopathological data, and the increasing pace of research on new treatments and clinical trials, and ever-changing guidelines for treatment with the advent of novel drugs and evidence, $\mathrm{Al}$ is the need of the hour. There are numerous publications and active work on Al's role in the field of oncology. In this review, we discuss the fundamental terminology of AI, its applications in oncology on the whole, and its limitations. There is an inter-relationship between Al, machine learning and, deep learning. The virtual branch of Al deals with machine learning. While the physical branch of Al deals with the delivery of different forms of treatment-surgery, targeted drug delivery, and elderly care. The applications of $\mathrm{Al}$ in oncology include cancer screening, diagnosis (clinical, imaging, and histopathological), radiation therapy (image acquisition, tumor and organs at risk segmentation, image registration, planning, and delivery), prediction of treatment outcomes and toxicities, prediction of cancer cell sensitivity to therapeutics and clinical decisionmaking. A specific area of interest is in the development of effective drug combinations tailored to every patient and tumor with the help of Al. Radiomics, the new kid on the block, deals with the planning and administration of radiotherapy. As with any new invention, $\mathrm{Al}$ has its fallacies. The limitations include lack of external validation and proof of generalizability, difficulty in data access for rare diseases, ethical and legal issues, no precise logic behind the prediction, and last but not the least, lack of education and expertise among medical professionals. A collaboration between departments of clinical oncology, bioinformatics, and data sciences can help overcome these problems in the near future.
\end{abstract}

DOI https://doi.org/ 10.1055/s-0041-1735577. ISSN 0971-5851.
(C) 2021. Indian Society of Medical and Paediatric Oncology. All rights reserved.

This is an open access article published by Thieme under the terms of the Creative Commons Attribution-NonDerivative-NonCommercial-License, permitting copying and reproduction so long as the original work is given appropriate credit. Contents may not be used for commercial purposes, or adapted, remixed, transformed or built upon. (https://creativecommons.org/ licenses/by-nc-nd/4.0/)

Thieme Medical and Scientific Publishers Pvt. Ltd., A-12, 2nd Floor, Sector 2, Noida-201301 UP, India 


\section{Introduction}

Intelligence is a unique characteristic of humans that differentiates them from other species. Inspired by the complex neuronal network and its functions in human beings, the seeds of artificial intelligence (AI) were sown in the 1950s. The term AI was first coined by John McCarthy in 1956. He described it as the making of intelligent machines by combining two different fields-science and engineering. Later, it expanded to a complex interaction between various other fields such as robotics, natural language processing, and machine learning.

\section{Fundamentals of artificial intelligence}

Artificial Intelligence $(\mathrm{AI})$ requires human intelligence and is performed by a computer. An algorithmic model is developed by humans (programmers)-a code instructing the computer step by step for necessary acting, reasoning, and learning.

Machine learning (ML) is an algorithm that can learn from data and act according to this knowledge without extensive prior programming. Here, a specific task can be performed based on particular patterns and inferences. It does not need any clear instructions or any programs. Based on "training data," a mathematical model is created and the computer can learn to make predictions. The ML model performs better with exposure to more data over time. The three main types of ML algorithms are:

(i) Unsupervised ML: It depends on finding patterns. Unlabeled data are given as an input, following which the system looks for patterns and classifies the data according to the identified patterns.

(ii) Supervised ML: It predicts algorithms based on past learning. Labeled data are already given as input; hence, the system categorizes various new given inputs based on what has been learned with old labeled data. One of the most commonly used forms of supervised learning is deep learning.

(iii) Reinforcement ML: It uses a system of reward and punishment for algorithmic training.

Deep learning (DL) is a subdivision of machine learning methods created on artificial neural networks. This model is built based on how a huge data/information is processed by the human brain. A well-designed and trained DL model can predict and perform classification tasks with more accuracy, which exceeds human expertise at times. In the field of medicine, AI can be divided into two branches: virtual and physical. $^{1}$

Virtual branch of AI: It is primarily based on ML, where mathematical algorithms help improve experience-based learning. The applications of virtual $\mathrm{AI}$ in medicine include electronic health record (EHR) systems and neural networkbased guidance in treatment decisions.

Physical branch of AI: It is the use of machines (robots) in physical form (macroscopic or microscopic) for assistance in performing daily tasks, surgeries, or delivery of chemotherapeutic drugs to a specific site.
(1) Carebots: These are advanced and sophisticated robots. They are used to assist surgeons during operation, as intelligent prostheses for handicapped people, and care of the elderly.

(2) Nanobots: They refer to miniature devices ( $\operatorname{size}=0.1$ $10 \mu \mathrm{m})$ in the form of switches, motors, shuttles, or cars carrying a particular drug or molecule. One such example is the targeted drug delivery using nanoliposomes by overcoming the permeation and diffusion barriers of the conventional therapeutic agents.

\section{Applications of $\mathrm{Al}$ in oncology}

AI has been and is being explored in various fields of oncology related to histopathology, imaging, radiation oncology, medical oncology, translational oncology, and clinical decision-making as depicted in -Fig. 1.

\section{Cancer Screening}

AI has found its vital place in the screening of cancers. Several convolutional neural network (CNN) models from the 2017 Kaggle Data Science Bowl demonstrated 80 to 95\% accuracy for lung cancer screening by evaluation of suspicious nodules in thoracic computed tomography (CT) films. ${ }^{2}$ In the same year, the digital mammography (DM) DREAM challenge by IBM, tested whether AI algorithms could be equal to or supplement the radiologists in interpreting mammograms. AI-based algorithms have reviewed 640,000 digital mammograms with $81 \%$ and $80 \%$ specificity and sensitivity, respectively. $^{3}$ Scott et al. assessed the performance of AI in predicting breast cancer from a large dataset of mammograms from both the USA and UK. ${ }^{4}$ The false-positive and false-negative rates reduced by $5.7 \%, 1.2 \%$ (USA, UK) and $9.4 \%$, $2.7 \%$, respectively. An independent study was done comparing AI algorithms and the radiologist's interpretation of screening mammograms for detecting breast cancer. The AI surpassed all human readers (six radiologists): the area under the receiver operating characteristic curve (AUROC) for the AI system was greater by an absolute margin of $11.5 \%$ than that for an average radiologist.

\section{Cancer Diagnosis}

Within the field of diagnostics, $\mathrm{AI}$ is gaining a pivotal role in accurate and speedy results.

\section{Clinical Images}

The CNN-trained algorithm using 130,000 skin images classified malignant lesions with higher sensitivity and specificity as compared with 21 dermatologists. ${ }^{5}$ A $94 \%$ sensitivity in polyp detection using colonoscopy images from 1,290 patients was observed with $\mathrm{CNN}{ }^{6}$

\section{Radiographic Imaging}

A study using a DL-based AI algorithm for the prediction of disease based on electronic health records (EHRs) of patient's clinical history, laboratory investigations, and imaging findings has been successful in this arena. In this, a novel framework called "deep patient" was developed based on a 

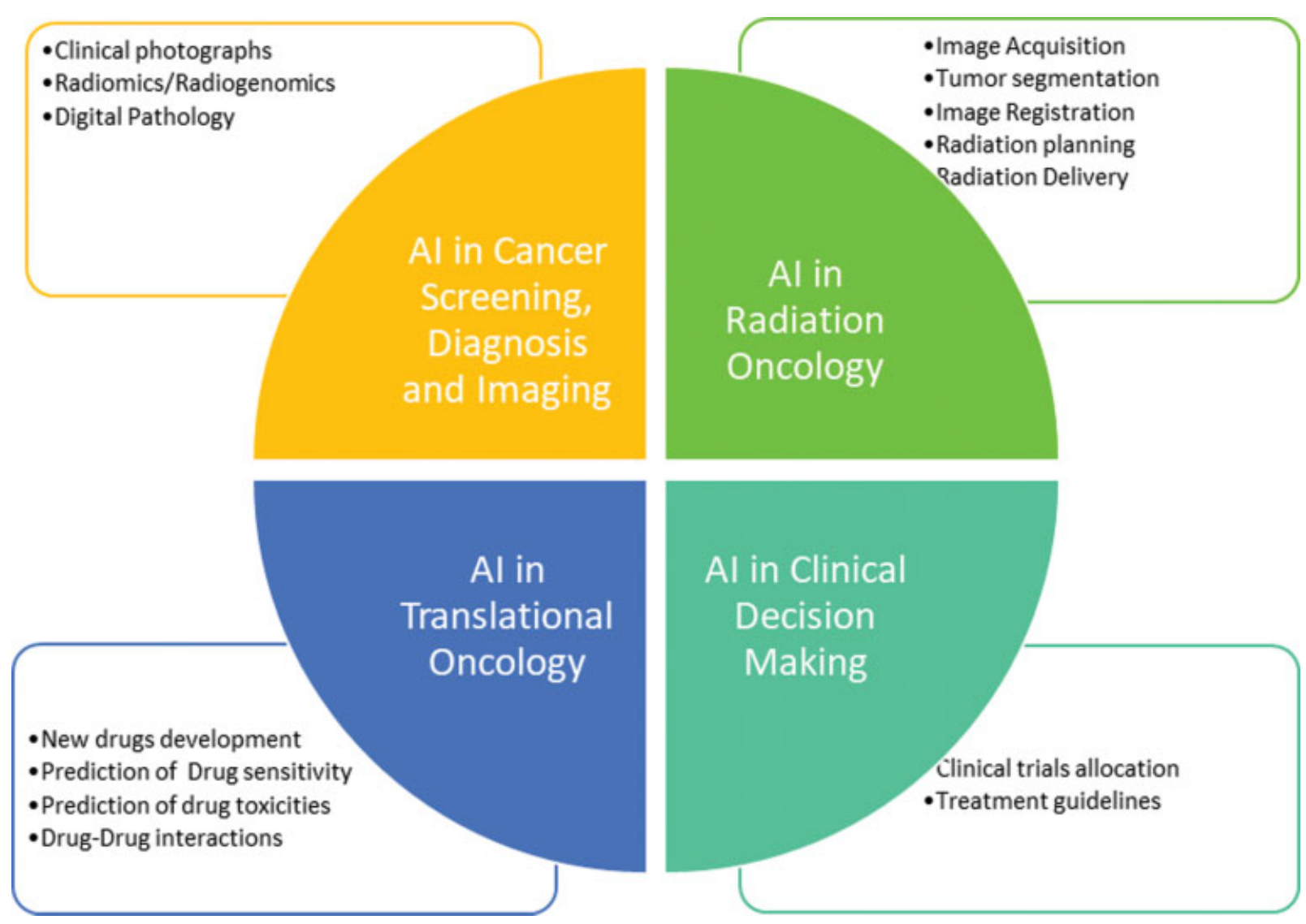

Fig. 1 Applications of artificial intelligence (Al) in oncology.

large dataset of patients' EHRs. Different patient sets with common patterns in the given data were identified. The new data were entered later and tested for its accuracy in predicting novel diseases in the next 1 year. It predicted the development of a variety of diseases including cancers of the prostate, rectum, and liver with $93 \%$ accuracy. ${ }^{7}$ Another CNN-based model showed $>85 \%$ accuracy in identifying extranodal extension (ENE) in head and neck cancers on diagnostic, contrast-enhanced CT scans. ${ }^{8}$

The CNNs have been able to predict both IDH mutation and MGMT methylation status with $85 \%$ to $95 \%$ and $83 \%$ accuracy, respectively, based on raw imaging data alone. ${ }^{9,10}$ A CNN model successfully predicted the complete response to neoadjuvant chemoradiation with $80 \%$ accuracy in locally advanced rectal cancer. ${ }^{11}$ Additionally, a radiomics signature using extracted features from CT data and an ML algorithm were able to predict underlying CD8 cell tumor infiltration and, remarkably, response to immunotherapy for a variety of advanced cancer patients in phase 1 trials treated with antiPD-1 or anti-PD-L1 monotherapy. ${ }^{12}$

\section{Digital Pathology}

The diagnostic rates of lymph node metastasis in resected specimens of carcinoma breast using DL CNN algorithms were found to be the same as those observed in a group of pathologists. ${ }^{13}$ Similarly, in adenocarcinoma of the prostate, a $75 \%$ rate of agreement of Gleason grading was seen between the DL algorithm and pathologists. ${ }^{14} \mathrm{~A}$ trained CNN was developed to predict six genetic mutations (STK11, EGFR, FAT1, SETBP1, KRAS, and TP53) from lung cancer biopsies. This paved the way for predicting a genotype or mutation based on histopathologic architectural patterns. ${ }^{15}$ These methods will be cost-effective as compared with tests for direct mutational analysis.

\section{Radiation Therapy}

Al has been proposed for image acquisition, tumor segmentation, image registration, radiation planning, and radiation delivery-the steps during treatment workflow in radiotherapy.

\section{Image Acquisition}

CT scan is the basic imaging for getting electronic density values. It helps in the planning of radiation therapy using algorithms for the calculation of doses. However, MRI is advantageous for better soft tissue delineation and acquisition of multi-planar images. There has been continuous research in the development of methods to generate a CT scan using MRI data-a synthetic CT scan (sCT). Of these methods, deep embedding CNN has shown promising results in terms of efficiency, time consumption, high-quality image resolution, and fewer artifacts. ${ }^{16}$ Algorithms for creating sCT from primary MRI images and for generation of radiation plan have been used in MRI only for prostate radiotherapy. ${ }^{17}$ 


\section{Segmentation of Tumors and Organs at Risk}

Manual contouring of organs at risk (OAR) and the volume of the target is a time-consuming task with huge interobserver variability. ${ }^{18}$ To overcome this hurdle, automatic contouring software using knowledge-based algorithms (atlas, machine learning, models based on statistical shape and appearance); region-based (adaptive thresholding, graph cuts, and contouring by watershed); or a combination of the knowledge and region-based have been used. ${ }^{19}$ In a recent study by Lustberg, ${ }^{20}$ the deep learning contouring outperformed the atlas-based contouring for the lungs and spinal cord. With deep learning methods, $79 \%$ of the median time was saved in comparison to manual methods. Men et al. ${ }^{21}$ hypothesized a new deep dilated CNN-based method for target auto-segmentation and volume delineation of OAR. It took on an average 45 seconds for one patient for segmentation of all clinical target volume (CTV) and OAR which is far less time than that taken for drawing the structures conventionally.

\section{Image Registration}

For image registration (to align an image to the reference image), mathematically applied transformations are used. The two traditional registration methods used in radiotherapy are the intensity-based method and the rigid method. In their review on newer techniques of image registration, Viergever et $\mathrm{al}^{22}$ found the deep learning process for registration of the images to be easier and user-friendly. This has been substantiated by studies done later by Yang et al. ${ }^{23}$ and Miao et al. ${ }^{24}$ using DL- and CNN-based methods, respectively.

\section{Radiation Planning}

The planning process for radiotherapy using AI algorithms was pioneered by McIntosh et al. ${ }^{25}$ They used a voxel-based method for predicting the dose and dose-mimicking method in planning radiation for head and neck cancers. The advantage of this adaptive radiotherapy planning process is not limited to saving time but also includes dose adjustments related to age, sex, race, and genetic makeup. ${ }^{26}$

PORTOS is the first clinical radiogenomics assay that uses predictive biomarkers to determine the tumor sensitivity to radiation. $^{27}$

\section{Radiation Delivery}

The recent advances in radiotherapy such as IGRT and IMRT focus more on image-guided delivery of radiation and less on the patient's position. Positional deviation leads to dose variation and hence compromises the efficacy of the treatment. Ogunmolu et al. ${ }^{28,29}$ developed a soft-robot actuator for head and neck radiotherapy without using a mask to monitor intra- and interfraction movements during radiation. It showed promising results in controlling the movement of the head to within the nearest $2 \mathrm{~mm}$ (millimeter) as compared with the trajectory of reference. Park et al. ${ }^{30}$ have used the data of breathing patterns for intra- and interfraction fuzzy deep learning (FDL) to decrease tumor-tracking time and adjust radiation doses in lung cancer patients according to breathing movements.

\section{Treatment Outcomes}

In 2003, an ANN analysis was done on 125 nonsmall cell lung cancer patients to predict their 5-year survival rates based on the immunohistochemical and clinicopathological variables. $^{31}$ The ANN-based prediction model was superior to logistic regression analysis in estimating the 5-year NSCLC survival rates ( $87 \%$ vs. $78 \%$ ). In-silico analysis using clinical and dosimetric data was also done to predict the genitourinary and lower gastrointestinal toxicity resulting from radiotherapy to prostate, ${ }^{32-34}$ hepatobiliary toxicity after liver irradiation, ${ }^{35}$ and rectal toxicity in cervical cancer patients receiving radiation therapy. ${ }^{36}$

\section{Translational Oncology}

The utility of DL neural networks has extended even to the field of translational oncology. With advancements in biotechnology and biochemistry, there is huge information on protein structure, and protein-protein and drug-protein interactions. The structure of a protein can be predicted. ${ }^{37}$ To speed up the drug development process, CNN and ML methods were used to predict the side effects of various drug combinations. $^{38}$ It saves time and financial resources by halting the drug development process due to serious toxicities. The cells can be characterized according to different mitotic stages ${ }^{39}$ based on microscopy images. The progenitor cell lineage in the future can be delineated based on their microscopic images. ${ }^{40}$ Deep learning artificial neural networks (DL ANNs) have been trained to predict the chances of failure of around 200 drugs based on transcriptomic response signatures. ${ }^{41}$ By combining both genomic and chemical characteristics, AI can predict the sensitivity of tumor cells to therapeutics. ${ }^{42}$ The role of AI has been extended even to immunologicals by predicting the peptide major histocompatibility complex (MHC) binding of the immunological drugs using CNNs. ${ }^{43}$

\section{Role of Al in Clinical Decision-Making}

With the enormous amount of ongoing research in oncology that includes clinical trials, new drug development, and the discovery of predictive biomarkers, there is a splendid opportunity for AI to assist in data synthesis and guide us in making and taking decisions for cancer patients. More commercial applications are being developed that utilize DL and natural language processing to utilize this huge data from bench-side to bed-side. ${ }^{44}$ International business machines (IBM) in collaboration with Watson for Oncology (WFO) used AI for linking the patient data to national treatment guidelines. The WFO demonstrated high concordance rates of the multi-disciplinary team recommendations with that of $\mathrm{AI}$ in breast cancer patients. ${ }^{45,46}$ However, the same IBM Watson cognitive computing system failed in taking the right decisions related to cancer treatment. After a huge expenditure of $\$ 62$ million, the joint venture of IBM and University of Texas MD Anderson Cancer Center for the development of an advanced Oncology Expert Advisor had to be called off! Retrospection showed training with a small dataset as a cause for this failure. 


\section{Role of $\mathrm{Al}$ in Hemato-Oncology}

Several CNN-based models have distinguished between different white blood cells on peripheral smear with more than 95\% AUROC scores. ${ }^{47}$ Similar work is underway for the interpretation of bone marrow specimens. In patients with lymphoma, automated analysis for bone marrow involvement has been successful. ${ }^{48}$ The risk stratification for advanced Hodgkin lymphoma based on PET-CT images was also tried with encouraging results. ${ }^{49}$

In malignant hematology, there are myriad clinical and laboratory-based prognostic scores for each cancer. However, there is a significant variation on a case-to-case basis. AI can deal with this complex data and help in personalized therapy. Upfront AML/MDS risk stratification can be improvised with the help of $\mathrm{AI}^{50}$ One of the most important poor prognostic factors after treatment completion in hematological malignancies is the detectable minimal residual disease (MRD). AI matched the human-level performance in MRD detection using flow cytometry in patients with acute myeloid leukemia. ${ }^{51}$ A retrospective data analysis by Sasaki et al in CML-treated patients showed a longer survival rate for ML-informed treatments. Algorithm-based models in MDS predicted the response to hypomethylating agents. ${ }^{52}$

\section{Role of AI in Resource-Limited Settings}

In developing countries such as India, there is a huge disparity between the numbers of patients and the numbers of health workers. A SWOT (Strengths, Weakness, Opportunities, Threat) analysis for this cutting-edge technology in developing countries is as follows:

Strengths: Availability of large amounts of data, emerging young population with a lot of talent and interest in this new technology, eco-friendly.

Weakness: High cost, lack of trained personnel, lack of standardization of data collection, storage and processing, data privacy, data security, digital divide (lack of access to high-speed broadband networks in rural areas).

Opportunities: Public-private partnerships, national and international collaborations, Government funding (NITINational Institution of Transforming India, DBT-Department of Biotechnology) for the development of $\mathrm{AI}$ in health care settings.

Threats: Loss of technological and health care workers' employment, legal and ethical issues.

\section{Limitations of Al}

This groundbreaking technology has its limitations that are hindering AI to be used in routine clinical settings.

First, the greatest hindrance is the external validation of DL applications and the generalizability of DL applications to all patients and tumors. The heterogeneous medical data across institutions and different patient populations will require multiple validation sets to prove the performance of AI. Second, access to data is a problem. It would be difficult to provide data for diseases with less prevalence. Contributing to this data scarcity, there are ethical and legal issues involved in sharing of protected information about patient's health across different institutions and also data heterogeneity along with incomplete data collection and competition between institutions. The use of findable, accessible, interoperable, reusable (FAIR) data, ${ }^{53}$ and many other opportunities are being provided for various research groups to address this issue. ${ }^{54}$ Third, tracing the exact logic behind the predictions of DL is a hilarious task. Hence, it is rightly called the "black box" problem, which means the explanation for how and why it gave the output based on the input given, cannot be provided by $\mathrm{AI}^{55}$ The era of personalized and evidence-based medicine demands a rationale for every clinical decision being taken. The specific characteristic(s) of the input data that contributed to the ultimate output cannot be pinpointed in DL algorithms. From the perspective of both practitioner and regulatory bodies, this interpretability is a challenge for adopting AI-based algorithms into the healthcare system. ${ }^{56,57}$ Fourth, AI in radiotherapy and pathology is mostly dependent on the images for autosegmentation, hence quality and the number of images used for training dataset matters a lot in giving the final output. Poor-quality images and fewer images lead to faulty assessments by AI. Fifth, medical professionals lack the education and expertise required for dealing with AI-related algorithms. A collaboration between departments of clinical oncology, bioinformatics, and data science is needed to tide over this problem. Lastly, being in an initial start-up stage, the cost will be higher and institutions need financial planning accordingly.

\section{Conclusion}

The breakthrough technology of this millennium "artificial intelligence" has a promising future in all the fields of oncology. It is a necessary tool for handling enormous clinical data, accurate delivery of treatment, improving personalized treatment selection, and predicting patient and disease outcomes.

Like any new invention, AI also has had and continues to have some hiccups, but more research into these areas can help address them. It is not surprising to say that in near future, AI will become a new "sixth sense" for every oncologist.

Conflict of Interest

None declared.

\section{References}

1 Hamet P, Tremblay J. Artificial intelligence in medicine. Metabolism 2017;69:S36-S40

2 Data Science Bowl. 2017 Accessed July 3, 2020 at: https://www. kaggle.com/c/data-science-bowl-2017

3 The digital mammography DREAM challenge. Accessed July 11, 2020 at: https://www.ibm.com/blogs/research/2017/06/dreamchallenge-results/

4 McKinney SM, Sieniek M, Godbole V, et al. International evaluation of an AI system for breast cancer screening. Nature 2020;577 (7788):89-94

5 Webster DE, Suver C, Doerr M, et al. The Mole Mapper Study, mobile phone skin imaging and melanoma risk data collected 
using ResearchKit. Sci Data 2017;4:170005https://doi.org/ 10.1038/sdata.2017.5

6 Wang P, Xiao X, Brown JRG, et al. Development and validation of a deep-learning algorithm for the detection of polyps during colonoscopy. Nat Biomed Eng 2018;2:741-748

7 Miotto R, Li L, Kidd BA, Dudley JT. Deep patient: an unsupervised representation to predict the future of patients from the electronic health records. Sci Rep 2016;6:26094https://doi.org/ 10.1038/srep26094

8 Kann BH, Aneja S, Loganadane GV, et al. Pretreatment identification of head and neck cancer nodal metastasis and extranodal extension using deep learning neural networks. Sci Rep 2018; 8:14036.https://doi.org/10.1038/s41598-018-32441-y

9 Chang K, Bai HX, Zhou H, et al. Residual convolutional neural network for determination of IDH status in low- and highgrade gliomas from MR imaging. Clin Cancer Res 2018; 24:1073-1081

10 Chang P, Grinband J, Weinberg BD, et al. Deep-learning convolutional neural networks accurately classify genetic mutations in gliomas. Am J Neuroradiol 2018;39:1201-1207

11 Bibault JE, Giraud P, Durdux C, et al. Deep learning and radiomics predict complete response after neo-adjuvant chemoradiation for locally advanced rectal cancer. Sci Rep 2018;8:12611. Doi: 10.1038/s41598-018-30657-6

12 Sun R, Limkin EJ, Vakalopoulou M, et al. A radiomics approach to assess tumour-infiltrating CD8 cells and response to anti-PD- 1 or anti-PD-L1 immunotherapy: an imaging biomarker, retrospective multicohort study. Lancet Oncol 2018;19:1180-1191

13 Bejnordi BE, Veta M, van Diest PJ, et al. Diagnostic assessment of deep learning algorithms for detection of lymph node metastases in women with breast cancer. JAMA 2017;318:2199-2210

14 Arvaniti E, Fricker KS, Moret M, et al. Automated Gleason grading of prostate cancer tissue microarrays via deep learning. Sci Rep 2018;8:12054, https://doi.org/10.1038/s41598-018-30535-1

15 Coudray N, Ocampo PS, Sakellaropoulos T, et al. Classification and mutation prediction from non-small cell lung cancer histopathology images using deep learning. Nat Med 2018;24:1559-1567

16 Arabi H, Dowling JA, Burgos N, et al. Comparative study of algorithms for synthetic CT generation from MRI: consequences for MRI-guided radiation planning in the pelvic region. Med Phys 2018;45:5218-5233

17 Siversson C, Nordström F, Nilsson T, et al. Technical note: MRI only prostate radiotherapy planning using the statistical decomposition algorithm. Med Phys 2015;42:6090-6097

18 Vinod SK, Jameson MG, Min M, Holloway LC. Uncertainties in volume delineation in radiation oncology: a systematic review and recommendations for future studies. Radiother Oncol 2016; 121:169-179

19 Hoang Duc AK, Eminowicz G, Mendes R, et al. Validation of clinical acceptability of An atlas-based segmentation algorithm for the delineation of organs at risk in head and neck cancer. Med Phys 2015;42:5027-5034

20 Lustberg T, van Soest J, Gooding M, et al. Clinical evaluation of atlas and deep learning based automatic contouring for lung cancer. Radiother Oncol 2018;126(02):312-317

21 Men K, Dai J, Li Y. Automatic segmentation of the clinical target volume and organs at risk in the planning CT for rectal cancer using deep dilated convolutional neural networks. Med Phys 2017;44:6377-6389

22 Viergever MA, Maintz JBA, Klein S, Murphy K, Staring M, Pluim JPW. A survey of medical image registration - under review. Med Image Anal 2016;33:140-144

23 Yang X, Kwitt R, Styner M, Niethammer M. Quicksilver: Fast predictive image registration-a deep learning approach. NeuroImage 2017 Sep 1;158:378-396

24 Miao S, Wang ZJ, Zheng Y, Liao R. Real-time 2D/3D registration via CNN regression. Paper presented at: Biomedical Imaging ISBI 2016 IEEE 13th International Symposium, IEEE 2016:1430-1434
25 McIntosh C, Welch M, McNiven A, Jaffray DA, Purdie TG. Fully automated treatment planning for head and neck radiotherapy using a voxel-based dose prediction and dose mimicking method. Phys Med Biol 2017;62:5926-5944

$26 \mathrm{Kim} \mathrm{KH}$, Lee S, Shim JB, et al. A text-based data mining and toxicity prediction modeling system for a clinical decision support in radiation oncology: a preliminary study. J Korean Phys Soc 2017; 71:231-237

27 Kang J, Rancati T, Lee S, et al. Machine learning and radiogenomics: lessons learned and future directions. Front Oncol 2018; 8:228. Doi: $10.3389 /$ fonc.2018.00228

28 Ogunmolu OP, Gu X, Jiang S, Gans NR. A real-time, soft robotic patient positioning system for mask-less head-and-neck cancer radiotherapy: an initial investigation. Paper presented at: IEEE International Conference Automation Science Engineering CASE; August 24-28; 2015; Gothenburg, Sweden; 2015:1539-1545

29 Ogunmolu OP, Gu X, Jiang S, Gans NR. Vision-based control of a soft robot for maskless head and neck cancer radiotherapy. Paper presented at: IEEE International Conference Automation Science EngIneering CASE; August 21-24; 2016; Fort Worth, United States 2016:180-187

30 Park S, Lee SJ, Weiss E, Motai Y. Intra-. and inter-fractional variation prediction of lung tumors using fuzzy deep learning. IEEE J Transl Eng Health Med 2016;4:1-12

31 Hanai T, Yatabe Y, Nakayama Y, et al. Prognostic models in patients with non-small-cell lung cancer using artificial neural networks in comparison with logistic regression. Cancer Sci 2003; 94(05):473-477

32 Pella A, Cambria R, Riboldi M, et al. Use of machine learning methods for prediction of acute toxicity in organs at risk following prostate radiotherapy. Med Phys 2011;38:2859-2867

33 Carrara M, Massari E, Cicchetti A, et al. Development of a ready-touse graphical tool based on artificial neural network classification: application for the prediction of late fecal incontinence after prostate cancer radiation therapy. Int J Radiat Oncol Biol Phys 2018;102(05):1533-1542

34 Lee S, Kerns S, Ostrer H, et al. Machine learning on a genome-wide association study to predict late genitourinary toxicity after prostate radiation therapy. Int J Radiat Oncol Biol Phys 2018;101:128-135

35 Ibragimov B, Toesca D, Chang D, et al. Development of deep neural network for individualized hepatobiliary toxicity prediction after liver SBRT. Med Phys 2018;45:4763-4774

36 Zhen X, Chen J, Zhong Z, et al. Deep convolutional neural network with transfer learning for rectum toxicity prediction in cervical cancer radiotherapy: a feasibility study. Phys Med Biol 2017;62:8246-8263

37 Wang J, Cao H, Zhang JZH, Qi Y. Computational protein design with deep learning neural networks. Sci Rep 2018;8:6349. Doi: 10.1038/s41598-018-24760-x

38 Zitnik M, Agrawal M, Leskovec J. Modeling polypharmacy side effects with graph convolutional networks. Bioinformatics 2018; 34:i457-i466

39 Eulenberg P, Köhler N, Blasi T, et al. Reconstructing cell cycle and disease progression using deep learning. Nat Commun 2017; 8:463. Doi: 10.1038/s41467-017-00623-3

40 Buggenthin F, Buettner F, Hoppe PS, et al. Prospective identification of hematopoietic lineage choice by deep learning. Nat Methods 2017;14:403-406

41 Artemov AV, Putin E, Vanhaelen Q, et al. Integrated deep learned transcriptomic and structure- based predictor of clinical trials outcomes. Accessed December 29, 2016. at: https://www.biorxiv. org/content/10.1101/095653v2

42 Menden MP, Iorio F, Garnett M, et al. Machine learning prediction of cancer cell sensitivity to drugs based on genomic and chemical properties. PLoS One 2013;8:e61318. Doi: 10.1371/ journal.pone.0061318

43 Han Y, Kim D. Deep convolutional neural networks for panspecific peptide-MHC class I binding prediction. BMC Bioinformatics 2017;18:585 https://doi.org/10.1186/s12859-017-1997-x 
44 Sennaar K. AI and machine learning for clinical trials: examining 3 current applications. Emerj - Artificial Intelligence Research and Insight. Accessed January 18, 2019 at: https://emerj.com/ai-sectoroverviews/ai-machine-learning-clinical-trials-examining-x-currentapplications/

45 Somashekhar SP, Sepúlveda MJ, Puglielli S, et al. Watson for Oncology and breast cancer treatment recommendations: agreement with an expert multidisciplinary tumor board. Ann Oncol 2018;29(2):418-423

46 Liu C, Liu X, Wu F, et al. Using artificial intelligence (Watson for Oncology) for treatment recommendations amongst Chinese patients with lung cancer: feasibility study. J Med Internet Res 2018;20:e11087. Doi: 10.2196/11087

47 Hegde RB, Prasad K, Hebbar H, Singh BMK. Comparison of traditional image processing and deep learning approaches for classification of white blood cells in peripheral blood smear images. Biocybern Biomed Eng 2019;39(02):382-392

48 Chandradevan R, Aljudi AA, Drumheller BR, et al. Machine- based detection and classification for bone marrow aspirate differential counts: initial development focusing on nonneoplastic cells. Lab Invest 2020;100(01):98-109

49 Milgrom SA, Elhalawani H, Lee J, et al. A PET radiomics model to predict refractory mediastinal Hodgkin lymphoma. Sci Rep 2019; 9(01):1322. Doi: 10.1038/s41598-018-37197-z
50 Nazha A, Komrokji RS, Meggendorfer M, et al. A personalized prediction model to risk stratify patients with myelodysplastic syndromes. Blood 2018;132(Suppl 1):793-793

$51 \mathrm{Ni} \mathrm{W}, \mathrm{Hu} \mathrm{B}$, Zheng C, et al. Automated analysis of acute myeloid leukemia minimal residual disease using a support vector machine. Oncotarget 2016;7(44):71915-71921

52 Nazha A, Sekeres MA, Bejar R, et al. Genomic biomarkers to predict resistance to hypomethylating agents in patients with myelodysplastic syndromes using artificial intelligence. JCO Precis Oncol 2019;3:1-11

53 Wilkinson MD, Dumontier M, Aalbersberg IJ, et al. The FAIR guiding principles for scientific data management and stewardship. Sci Data 2016;3:160018https://doi.org/10.1038/sdata.2016.18

54 Chavan V, Penev L. The data paper: a mechanism to incentivize data publishing in biodiversity science. BMC Bioinformatics 2011; 12(Suppl 15):S2https://doi.org/10.1186/1471-2105-12-S15-S2

55 Castelvecchi D. Can we open the black box of AI? Nature 2016;538 (7623):20-23

56 Key Changes with the General Data Protection Regulation EUGDPR Accessed December 3, 2018 at: https://ec.europa.eu/ info/law/law-topic/data-protection/data-protection-eu_en

$57 \mathrm{Yu} \mathrm{KH}$, Beam AL, Kohane IS. Artificial intelligence in healthcare. Nat Biomed Eng 2018;2:719-731 